\section{Electron Tomography in the Study of Bacterial Structure and Function}

\section{Kenneth H. Downing, ${ }^{*}$ Haixin Sui, ${ }^{*}$ Luis R. Comolli* and Hoi-Ying Holman ${ }^{* *}$ \\ ${ }^{*}$ Life Science Division, ${ }^{* *}$ Earth Sciences Division, Lawrence Berkeley National Laboratory \\ khdowning@lbl.gov}

Bacteria contain a wealth of mechanisms that organize their internal and external components into a highly polar structure, frequently with distinctive shape, and constrain certain metabolic functions to particular parts of the cell. For example, cell division generally takes place at the middle of the cell, and a host of interacting proteins are involved in ensuring that the division site is positioned properly. Thus, in spite of the lingering perception among some scientists that these cells are simply bags of freely diffusing enzymes, there is much to be learned from ultrastructural studies. Light microscopy has given evidence of cytoskeletal components that presumably establish and maintain cell shape and participate
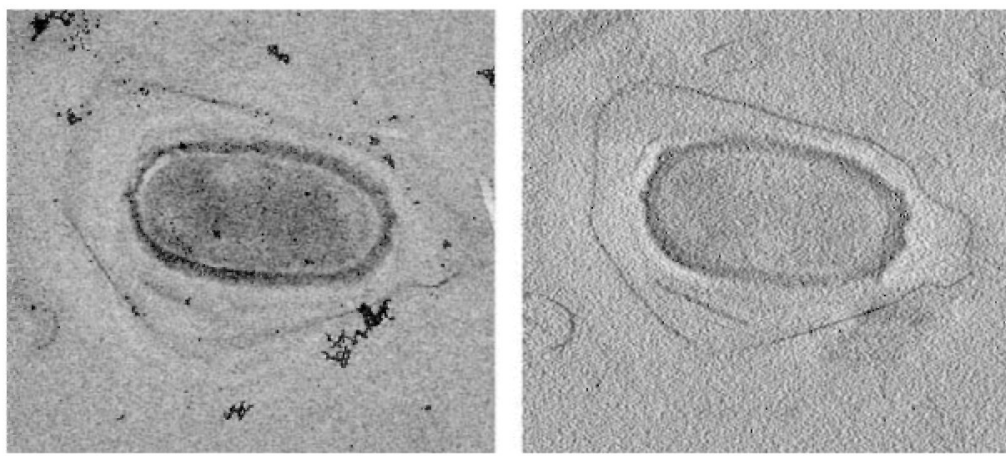

Figure 1. Unstained plastic section of Arthrobacter oxydans, a chromiumsequestering bacteritu. Left: A micrograph of the section shows the central part of the cell surrounded by a thick, low-density polysaccharide layer; width of the image is $2.5 \mu \mathrm{m}$, and the black spots are gold particles added to the surface to aid in image alignment. Right: A thin slice through the 3-D reconstruction shows the sheet-like structures that form in the presence of chromium outside of and within the polysaccharide layer.

in cell division. There is also much information about cell-cycledependent movement and localization of regulatory proteins in addition to specific locations for proteins involved in nucleic acid replication and construction of flagella, pili and attachment stalks, which are often found just at the ends of the cell. ${ }^{1}$ Electron microscopy offers the possibility to localize these components at a resolution that would allow us to achieve a far better understanding of their structures and interactions. A full understanding, though, requires that the structures be resolved in three dimensions as a realistic model of the original cell. The application of electron tomography can provide such a three-dimensional (3-D) model.

Tomography, as practiced in fields such as medicine, industrial inspection and electron microscopy, consists of obtaining a three-dimensional representation of an object from a set of projection images. In the electron microscope, the projections are taken as a tilt series. A computational operation called a "back projection" is then used to create a 3-D model of the sample. Over the last decade, electron tomography has developed into a productive and popular technique in biology and, more recently, material science. It has been used to elucidate many subcellular features that would not be evident from examination of thin sections. Iruou wunmonly, work is done with embedded and sectioned material that is cut thicker than would be used in conventional EM. In some cases, it is also possible to work with intact, frozen-hydrated cells preserved in their native state without staining or sectioning. Here we discuss some of the potentials and issues involved in the technique.

The ability to obtain a 3-D view of a specimen presents many opportunities, but also poses challenges in the tradeoffs that exist between using a thick specimen in order to obtain as great a volume as possible and resolution limitations that become more severe as the thickness increases. One result is that the mathematics of image contrast formation and back projection do not fit any of the simple approximations used in other aspects of electron microscopy. However, the resolution and interpretability of the reconstructions are generally limited by factors other than the failure of these mathematical approximations. Radiation damage sets a limit to the signal-to-noise ratio, and thus the effective resolution, in the case of frozen-hydrated samples, while effects of preservation and staining generally set the limits with embedded specimens.

It has been predicted that the ultimate resolution limit is around $\sim 20$ - $30 \AA$, for tomographic studies of moderately thick, frozen-hydrated samples. ${ }^{2}$ At this resolution we should be able to identify the shape and size of the major macromolecular complexes within a cell. Our long range goal, in fact, is to map out the locations of these complexes in order to gain understanding of their presence and interactions through the cell cycle. 3-D reconstructions of whole cells should lead to the ability to understand and even model spatial and temporal relationship between sub-cellular structures, organelles and macromolecular complexes through the full cycle of the cell, along with morphological changes, transport and specific localization of macromolecular complexes, and other dynamic events that regulate the life functions of these cells. While some exciting and promising results have been shown that represent progress toward these goals, a number of advances in instrumentation are still needed to make it routinely possible to reach the resolution required to identify particular proteins.

Achieving a specific resolution in a reconstruction requires a certain number of images in the tilt series, and this number in-

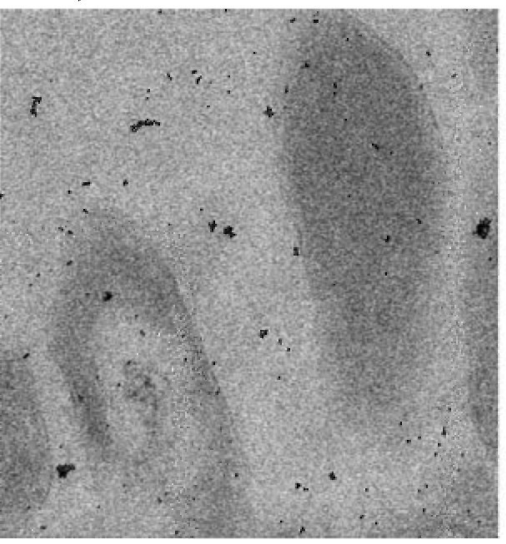

Figure 2. A plastic-embedded section of Desulfiovibrio vulgaris prepared after brief exposure to air. Left: A micrograph of the section, which was about $300 \mathrm{~nm}$ thick, shows two cells, one at the left cut in cross section and one on the right cut nearly longitudinally. Right: A slice through the 3-D reconstruction shows partially condensed nucleic acid and non-staining region toward the middle of the cell surounded by a denser region with a distincive structure.

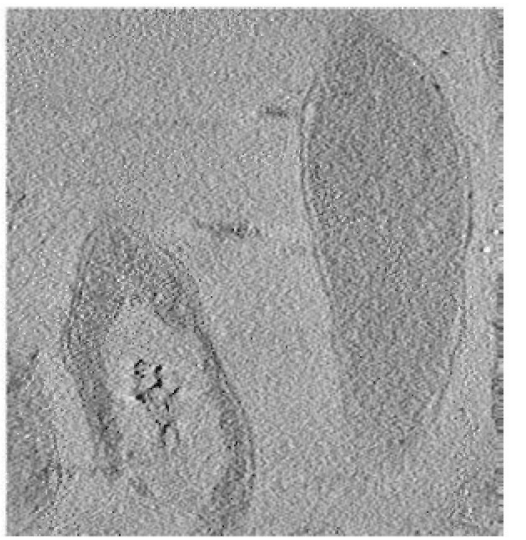



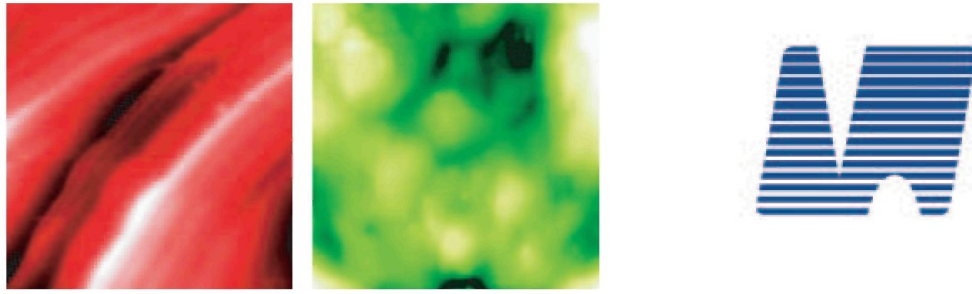

\section{Nano-R ${ }^{\mathrm{TM}}$ Brystal Soanner ${ }^{\mathrm{TM}}$}
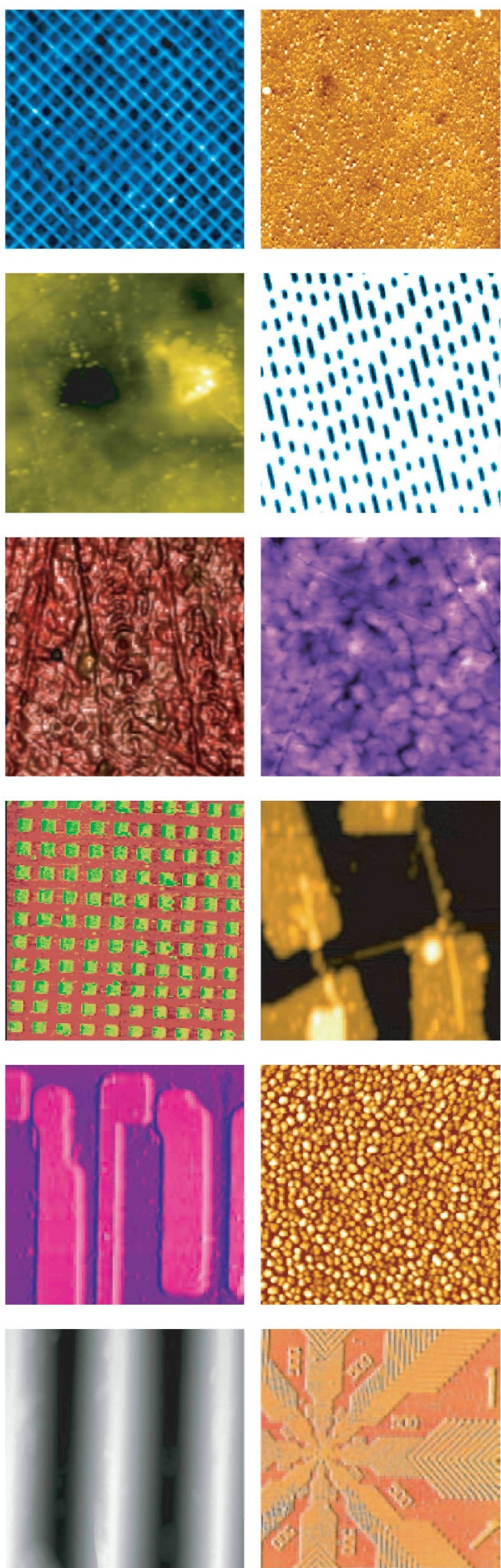

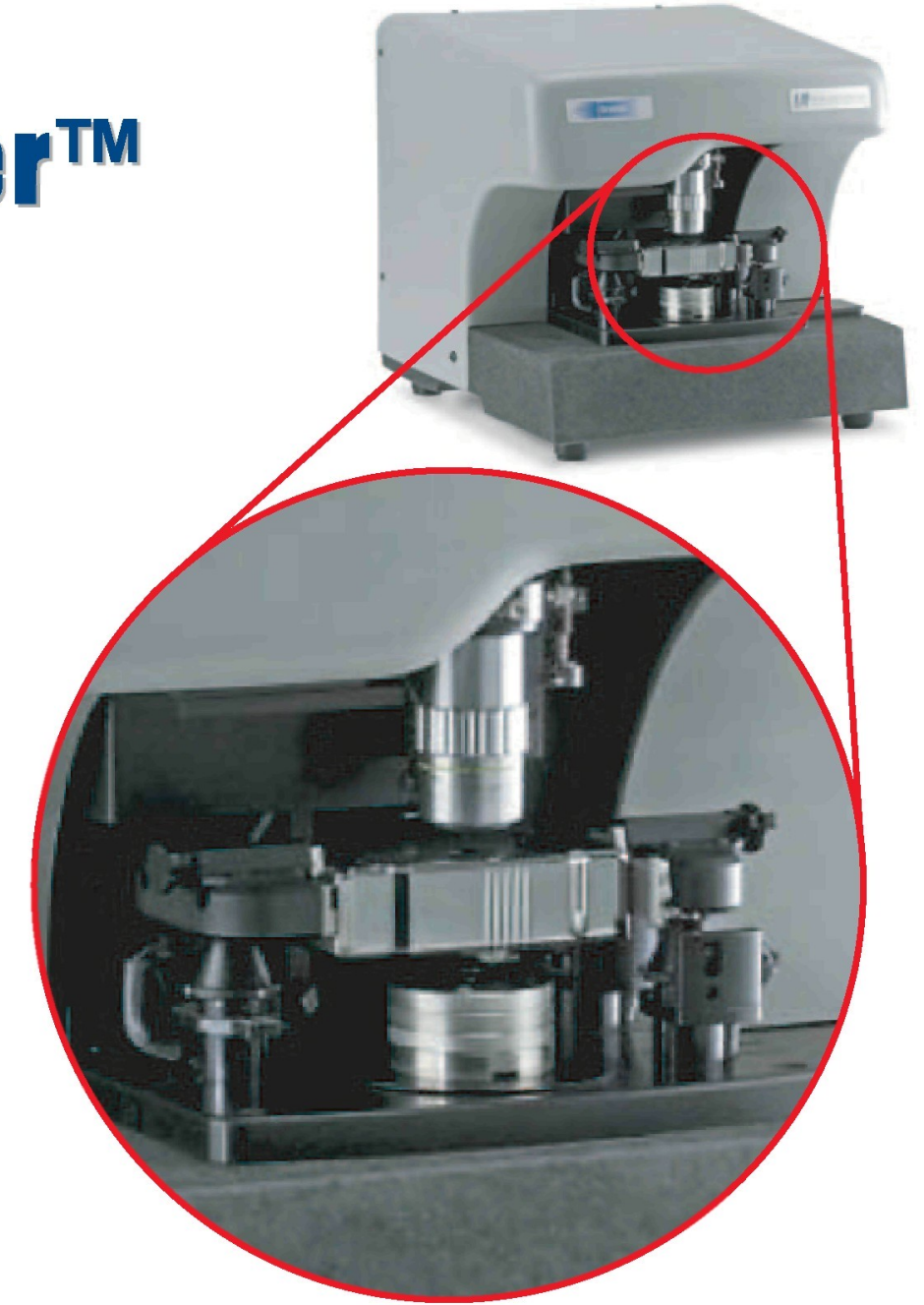

- Nano-R ${ }^{\mathrm{TM}}$ has a new scanner which makes topographic imaging simple

- Crystal Scanner uses Point \& Scan ${ }^{\text {TM }}$ technology load the sample, the probe and just scan

- No complex operating procedures - no laser to adjust

- Motorized x,y stage and automated video microscope features make high resolution nanoscale imaging easy

(800) 246-3704

3350 Scott Blvd. \#29 • Santa Clara, CA 95054

Phone: (408) 982-9492 - Fax: (408) 982-9151

www.pacificnano.com • www.probestore.com 
Figure 3. Frozen-hydrated (unstained) Magnetospirillum magnetotacticum. Left: The zero-degree tilt image from the series of micrographs, showing the shape of the entire cell which is about $2 \mu \mathrm{m}$ long. The chain of magnetosomes runs through most of this length. Center: An isosurface view of the 3-D reconstruction shows the magnetosome chain, which curves out of the plane of the specimen. The cell membranes also show up at this contour level, but appear discontinuous due to the noise level. Right: Slice through the reconstruction near one end of the cell shows a number of interesting features of the cell.

creases along with the specimen thickness. Especially for the case of frozen-hydrated samples, there is a limit to the total exposure that the specimen can tolerate without excessive beam damage. This total exposure has to be distributed among all the images of the tilt series, but at the same time each image needs to be taken with a sufficient exposure to provide enough signal for processing the images. Improving CCDs or other devices for image recording, optimizing the thickness and radiation tolerance of the specimens,
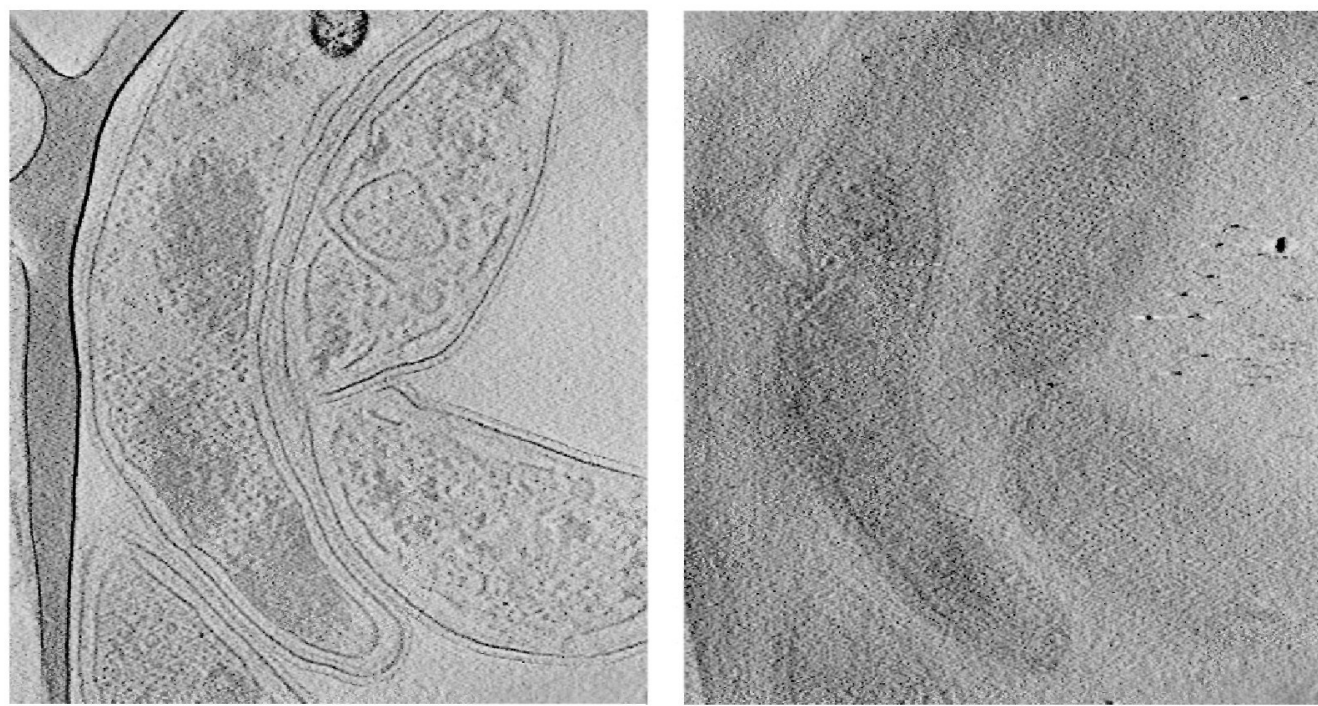

Figure 4. Two slices, at different heights, through the 3-D reconstruction of a frozen-hydrated sample of Caluobacter crescentis. The cell on the right is about to finish dividing. At the far left is part of the lacy carbon film used to support the sample. In the left image, which is a slice through the middle of the cells, a wealth of subcellular structure can be seen representing the distribution of ribosomes and other protein complexes, membranes and nucleic acids. These cells appear to have suffered some damage in the preparation process which makes these internal details easier to see, but this image gives a clear impression of the level of detail one can expect to achieve. On the right, a slice that grazes the top of the cells shows the periodic S-layer characteristic of these bacteria. and minimizing exposure during data recording will substantially improve the data. The use of an energy filter to limit effects of inelastic scattering also can improve the data greatly. Being able to tilt the grid around orthogonal axes to generate a two-axis tilt series makes a dramatic improvement in the quality of the results, but requires a type of specimen holder that is not yet widely available. All of these advances are being worked on and are at various stages of development in various labs, so it should not be long before they can be brought together to bring us close to the ultimate resolution limit.

In the meantime, much is being learned from studies of frozen hydrated samples as well as embedded and sectioned material. Staining inherently limits the resolution, but can provide a wealth of information not easily obtainable with unstained samples. Sectioning allows us to deal with cells that are too thick to work with intact, which includes a fair fraction of the interesting bacteria.

Much of our current work involves understanding processes in bacteria that might eventually be adapted for environmental cleanup and other efforts within the Department of Energy. One application has been with Arthrobacter oxydans, a bacterium that sequesters chromium by precipitation around the cell surface. These bacteria are too thick to study intact in frozenhydrated preparations but serve as an 
interesting example of what can be gained with embedded sections on such a small scale. Figure 1 shows an image of one of the cells that was grown in the presence of chromium, and a slice from the $3-\mathrm{D}$ reconstruction. The tomogram complements and extends information inferred from thin section TEM and whole-mount SEM concerning the chromium deposition in sheets that are both on the surface and discontinuously distributed throughout the polysaccharide layer that surrounds the cell. Modeling of these structures within the tomographic volume provides information that would be very difficult to obtain from work with thin sections.

Another example of a conventionally prepared bacterium is shown in fig. 2. This specimen is Desulfovibrio vulgaris, a bacterium that was once thought to require anaerobic conditions to survive but has since been found to be able to adapt to an oxygencontaining atmosphere. The cells undergo a number of distinctive morphological changes in the process of this adaptation, which include rearrangement of the nucleic acid, presumably in response to the need to produce a new set of proteins, and the production of a thick polysaccharide capsule that may inhibit oxygen entry into the cell. Comparing cells at various times after exposure to air gives us a catalog of these changes that complements studies of gene expression.

While the cells shown in figs. 1 and 2 are too thick to examine intact, a number of bacteria are thin enough to study at higher resolution in frozen-hydrated samples. We have studied several of these, including small elongated bacteria such as Magnetospirillum and Caulobacter. Figure 3 shows one example of frozen-hydrated Magnetospirillum magnetotacticum, a bacterium that precipitates iron into specifically oriented magnetic particles called magnetosomes that aid the cell in oriented swimming along an oxygen gradient. This cell has a chain of magnetosomes that extends through nearly the entire cell and is easily visible in both the original images and the reconstruction. In addition, many other features of the cell can be seen, especially in the membrane and cell wall, even at this low magnification. Of particular interest are the cytoskeletal elements that organize the magnetosome chain.

Much has been learned about the biochemical processes that regulate bacterial morphogenesis from studies of Caulobacter crescentis, a bacterium that has a series of distinctive stages in its cell cycle. Figure 4 shows images of cells at different stages and gives a sense of the type of information that will eventually be obtained by mapping out the structures of microbes in three dimensions and the locations of molecular complexes within them.

The range of environmental adaptations and related structural features and metabolic variations in the prokaryotic world is truly astounding. It is clear that electron tomography, applied over a wide resolution range, will play an increasingly important role in elucidating the molecular interactions, mechanisms and functions employed in this highly divergent class of organisms.

\section{References}

1. Shapiro, L., H.H. McAdams and R. Losick, Generating and exploiting polarity in bacteria, Science, 2002. 298 1942-1946.

2. Bohm, J., A.S. Frangakis, R. Hegerl, S. Nickell, D. Typke and W. Baumeister, Toward detecting and identifying macromolecules in a cellular context: Template matching applied to electron tomograms. Proc. Nat. Acad. Sci. USA, 2000. 97 14245-14250.
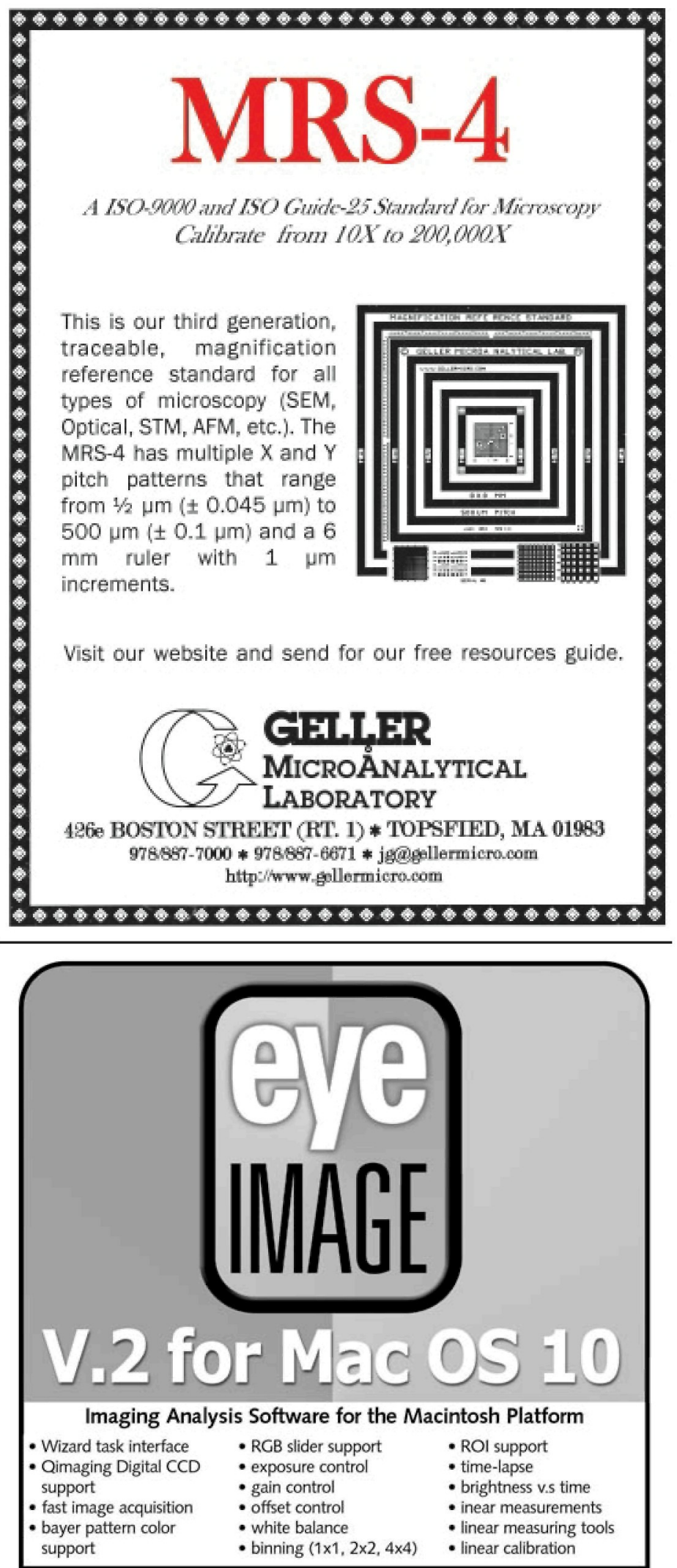

FREE electronic upgrades for 2 years

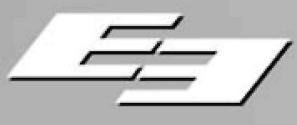

E\」 $P$ \X

www.empix.com 